\title{
Beard hair transplant to the hairline
}

\author{
Viroj Vong, MD \\ H.H.H. Natural Hair Transplant Center, Bangkok, Thailand
}

\begin{abstract}
In the present case report, an adult man underwent three separate hair transplantation procedures over a course of 5 years, followed by one tattoo procedure 2 years after completion of hair transplantation, to conceal and prevent hair loss. Still unsatisfied with his hairline and the unnatural look of the tattoo, the man returned to discuss options for another operation. After doing so, he decided to undergo an alternative hair transplant surgery whereby 1,750 follicular units were removed from his beard (below the jaw line) and transplanted into his temple; the procedure both esthetically improved the patient's hairline and camouflaged the tattoo.
\end{abstract}

Keywords: beard hair transplant; Follicular Unit Extraction; scalp micropigmentation; tattoo; baldness; hairline

\section{Introduction}

A 39-year-old Caucasian man presented first time in our clinic with baldness at the temple area and a receding hairline. He underwent three separate hair transplantation procedures at a competing hair transplant clinic to combat his hair loss. Importantly, these surgeries depleted a large amount of hair follicles from the posterior scalp: the prime donor site area for hair transplant patients.

The patient was dissatisfied with the results and decided to undergo another procedure-the medical hairline tattoo (also known as scalp micropigmentation; SMP)—on his temple area to cover up his receding hairline. Unfortunately, the patient was displeased with the outcome of the medical hairline tattoo as well, because it looked artificial, and he wanted a naturallooking hairline.

The patient consulted H.H.H. Natural Hair Transplant Center to camouflage the medical hairline tattoo. He decided to have a fourth hair transplant, wherein the hair follicles of his beard hair would be transplanted into his temple area. Thus, the patient underwent Follicular Unit Extraction (FUE) surgery, wherein 1,750 follicular units were harvested below the jawline (donor site) and then implanted into the temple area (recipient site) and frontal hairline; each follicular unit in the beard is typically composed of 1-3 hairs.

\section{Materials and methods}

The patient received local anesthesia to reduce pain and discomfort during surgery. Specifically, he was given $0.5 \% \mathrm{Li}$ docaine mixed with $0.125 \%$ Marcaine and Tumescent Fluid (1L Normal Saline Solution, $50 \mathrm{~mL}$ 1\% Xylocaine, $1 \mathrm{~mL}$ 1:1,000 adrenaline).

The attending surgeon then carefully drew the Line Thai Micro Hairline [1] at the recipient area (Fig. 1); this is an Ancient Thai technique for creating natural-looking hairlines.

The surgeon used a micro-surgical knife (Micro slit blade) to make 1,750 incisions-each of which was $1 \mathrm{~mm}$ in length and formed a hair canal into the temple. Next, the surgeon selected, and removed one-by-one, 1,750 follicular units that were locat- 
ed below the jawline using a sharp, 1-mm follicular unit extractor.

Photographs were captured to compare and analyze before and after results. Fig. 2 shows the beard follicular units and the occipital follicular units of an unrelated patient. The scalp hair follicle exhibits a thicker dermal layer while the beard hair follicle displays a thinner dermal layer, larger bulb, thicker shaft, with enhanced curling. Further, beard hair follicles are relatively easier to extract than scalp hair follicles. Fig. 3 shows the patient's beard (donation site) 1 day after the FUE surgery. Fig. 4 depicts the patient's left hairline, 1 year following transplantation surgery.

All of the follicular units were counted and prepared for implantation. To protect each hair follicle, they were placed into a platelet-rich plasma (PRP) solution, which stimulates hair growth and promotes healing. Proper maintenance and storage

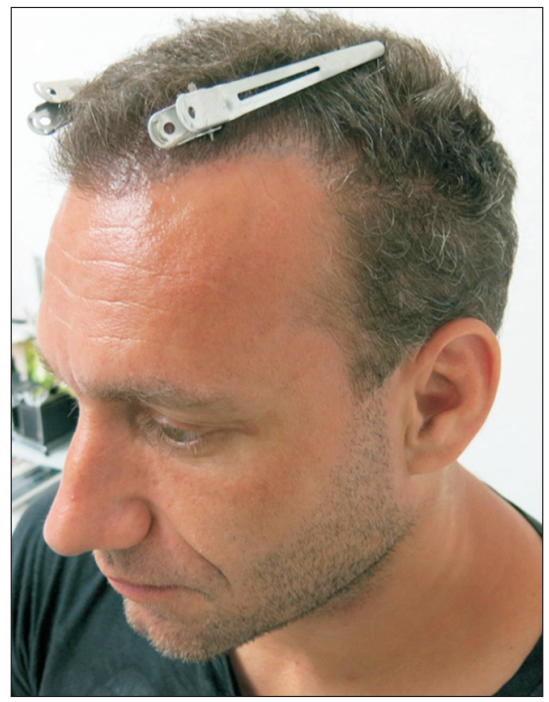

Fig. 1. Tattoo marks before surgery (left hairline). was essential during this step. The follicular units were hydrated and kept in containers at $4^{\circ} \mathrm{C}$.

The follicular units were placed carefully into the hair canals using micro-surgical forceps. This step was repeated until the entire recipient area was completed. The hair canals healed in 7 days.

\section{Post-operative care}

The patient took antibiotics capsule and painkillers by mouth before he left the clinic; he returned the next day for his first shampoo, and at this point he received instructions about how to wash the transplant area without damaging the new follicular units. Subsequently, the patient shampooed 3-4 times a day until all the scabs (dry blood on the scalp) had fallen out; this

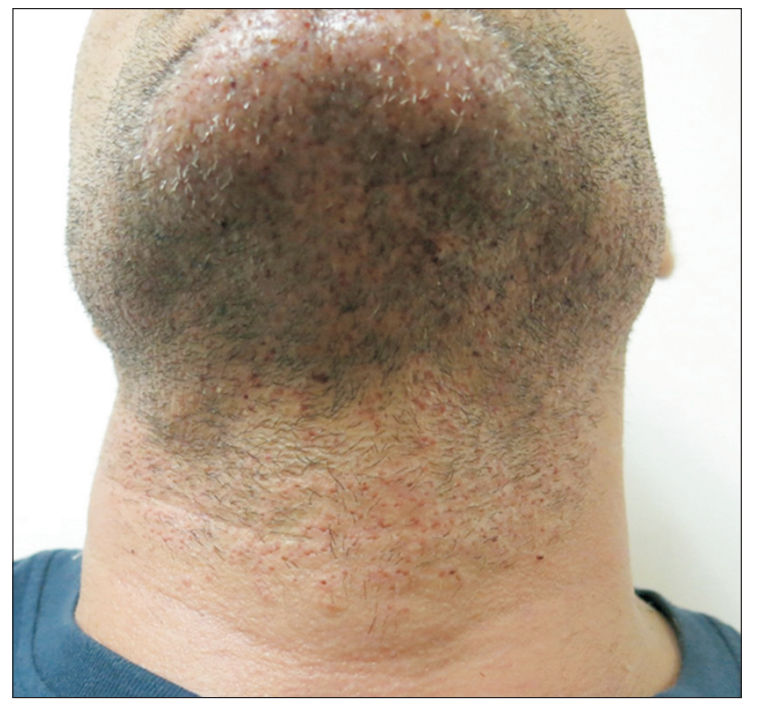

Fig. 3. Patient's beard (donation site) 1 day after Follicular Unit Extraction (FUE) surgery.

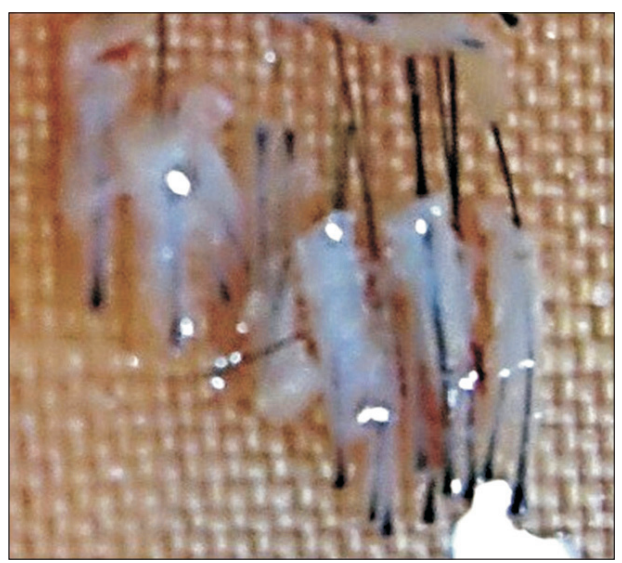

F.U.E scalp hair follicles

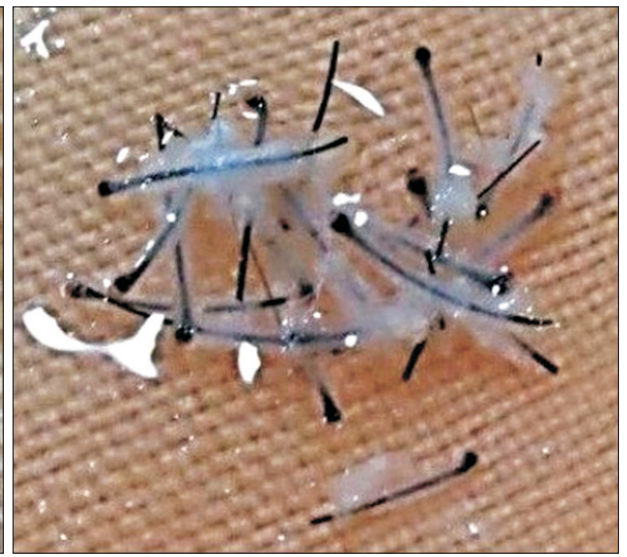

F.U.E beard hair follicles
Fig. 2. Comparison of beard and scalp hair follicular morphology. 
usually takes 1 week.

Furthermore, he was advised that he may experience swelling of the forehead for 2-7 days, that he should wait 2 weeks before shaving his beard, and that he should avoid direct sunlight on the transplant site for a minimum of 3 months.

In 3 months, new hair had started to grow, and 6 months after surgery, $75 \%$ of the transplanted hair had grown.

\section{Results}

After one year, the patient was very happy with his new, thick, natural-looking hairline. The tattoo mark was not visible, and the patient looked younger, stronger, and more handsome than he had done before surgery. Fig. 5 shows a comparison of the

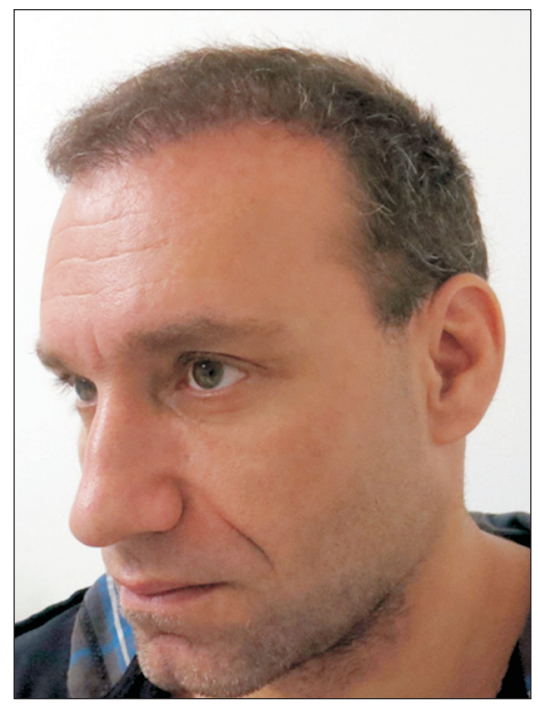

Fig. 4. Patient's left hairline 1 year following surgery. right hairline before surgery with that 1 year after. The patient's beard (donation site) 1 year after the FUE surgery is shown in Fig. 6.

\section{Discussion}

Beard hair (below or above the jawline) is an excellent potential source of hair follicles for a hair transplant, because (1) facial hair is thick in some men, (2) beard hair grows quickly, and (3) telogen occurs more frequently in the beard hair; this is the final phase of three in the process of hair loss, wherein the follicle lies dormant and can last from 3 months-3 years. The anatomy of a beard hair is shown in Fig. 7.

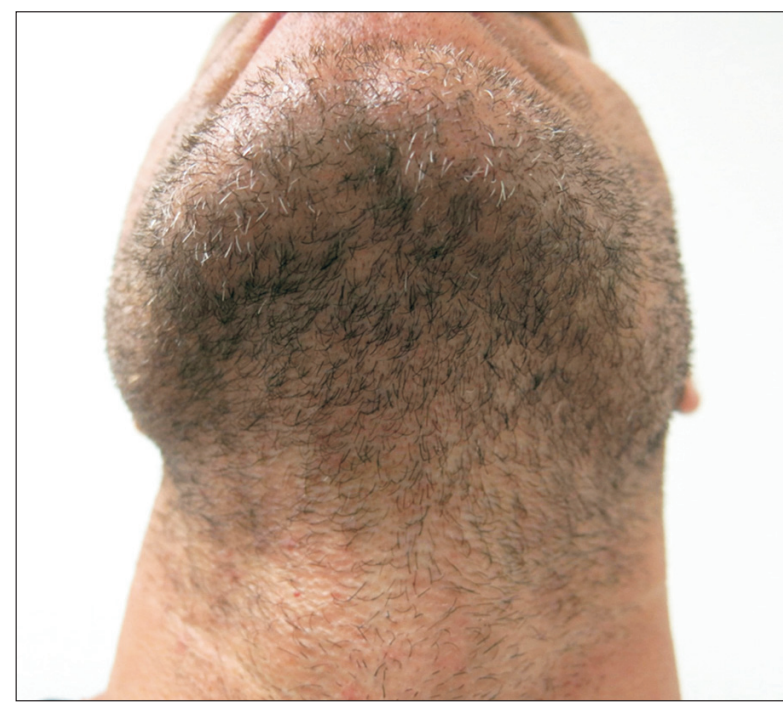

Fig. 6. Patient's beard (donation site) 1 year after the Follicular Unit Extraction (FUE) surgery.
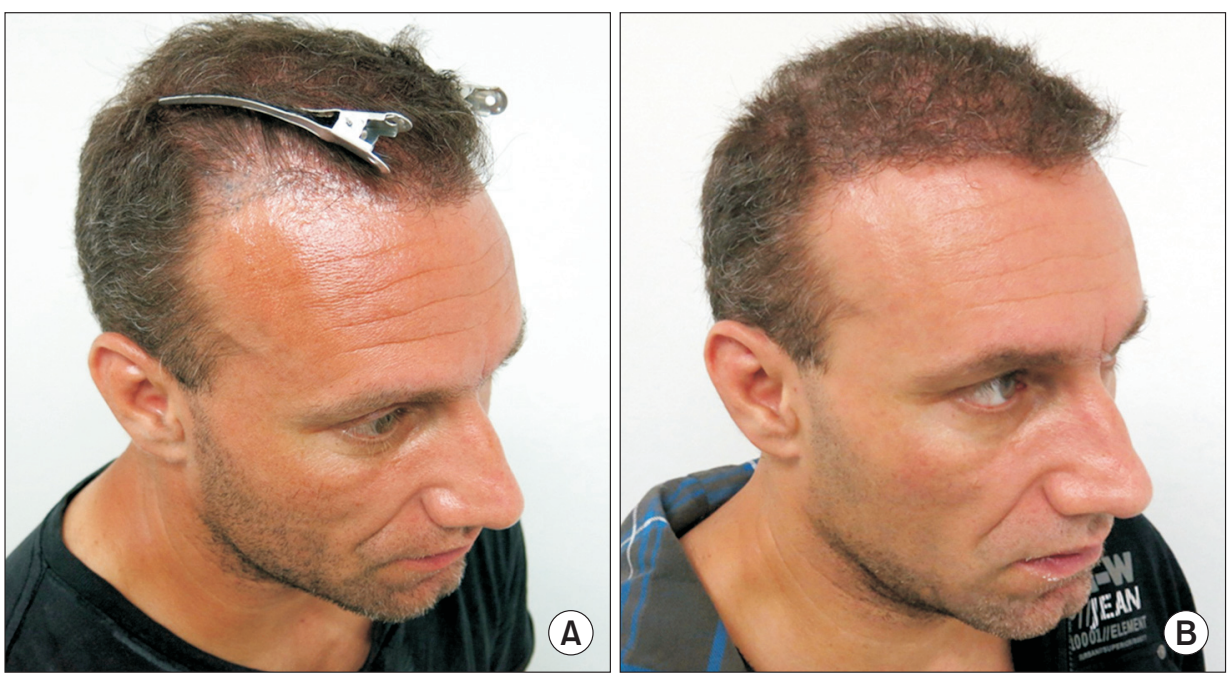

Fig. 5. Comparison of the right hairline (A) before and (B) one year after surgery. 


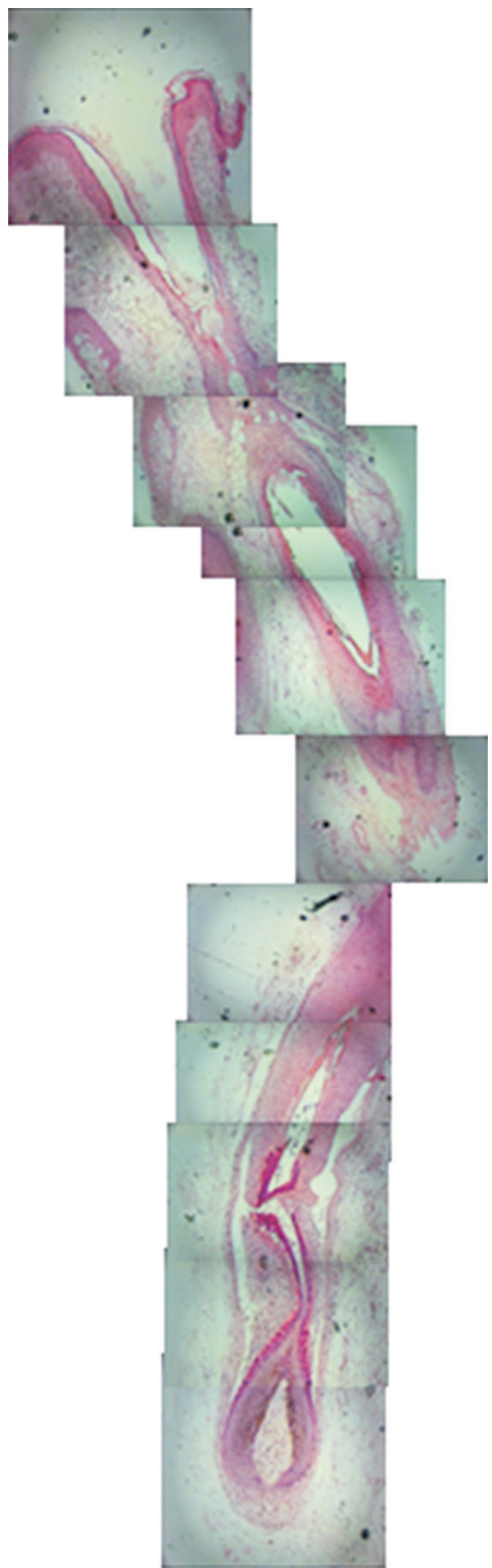

Fig. 7. Anatomy of beard hair.

Beard hair is thickly twisted and curly from the exit out, while the skin on beard area is thin, and therefore easier to extract than scalp hair using the extractor. In a practical sense, FUE

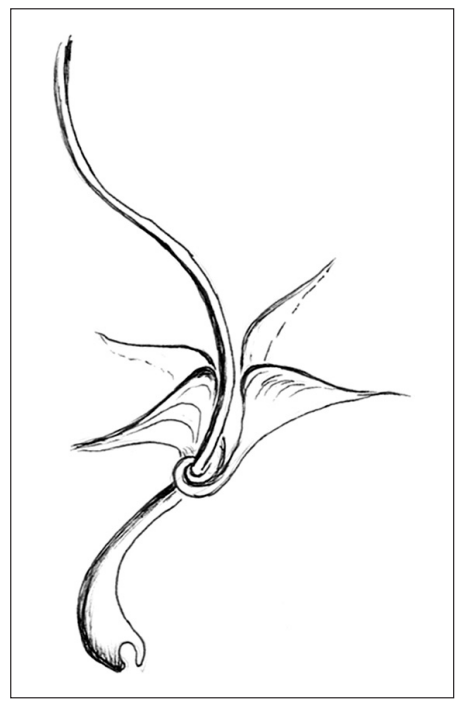

Fig. 8. Graphical depiction of the four erector pili muscles attached around the hair follicle and inserted into four skin creases.

is separate attachment of the four erector pili muscles (Fig. 8) from the hair follicle.

Dihydrotestosterone (DHT) is often associated with hair loss, why target the beard zone?

Indeed, DHT is a culprit for hair loss on the head. Ironically though, the hormone acts positively on the face, in that it causes growth of facial hair; therefore, the latter exhibits a lower risk of falling out after transplant. It is for this reason that beard hair is a viable source for hair transplants.

Who is the ideal choice to perform a beard hair to scalp hair transplant; an experienced hair surgeon or a robot?

This type of delicate surgery (beard hair to scalp hair transplant) is best performed by an experienced and skilled hair surgeon. The "human touch" is special and irreplaceable. Therefore, robotic hair transplant systems cannot compare to or replace the work of a surgeon on the highly sensitive jawline.

Tattoos (SMP) are one of a number of effective choices for treating baldness, thin hair, and scalp scars. However, the effect of SMP only functions on 2-dimensional skin, while the effect of hair is 3-dimensional: skin and above skin. Thus, to look natural, SMP must be performed in a hairy area, not on hairless skin or close to the edge of the hairline. Furthermore, surgeons perfoming SMP should use an ink that is the same color as the hair, but 30\%-40\% lighter; they should not use sharp dots, but rather very blurred dots, and uneven and random patterning is more natural. 


\section{Conclusion}

Beard hair (below or above the jawline) is a feasible follicular unit donor source area for hair transplants when the patient has undergone multiple hair transplants and exhausted the hair follicles present on the posterior scalp (optimal donor zone).

\section{Acknowledgements}

The author extends his most sincere thanks to both Bernard Koire MD and William R. Rassman MD for their persistent mentorship in Cosmetic and Hair transplant surgery. The au- thor further wishes to thank the patient for permitting the use his photos for publication.

\section{Conflicts of interest}

The author has nothing to disclose.

\section{Reference}

1. Vong V. Line thai micro hairline. In: Bloggs JJ, editor. Hair transplant forum international. Geneva: Natural Hair Transplant Center; 2008. p. 48. 